\title{
UNA NUEVA ESPECIE DE ECHEVERIA (CRASSULACEAE) DEL ESTADO DE TAMAULIPAS, MÉXICO
}

\author{
José Guadalupe Martínez-Ávalos \\ Arturo Mora-Olivo \\ Instituto de Ecología y Alimentos \\ Universidad Autónoma de Tamaulipas \\ 13 Blvd. López Mateos 928 \\ 87040 Cd. Victoria, Tamps. \\ jmartin@cactus.uat.mx \\ amorao@cactus.uat.mx
}

\begin{abstract}
RESUMEN
Se describe Echeveria rodolfi como una nueva especie para la flora del estado de Tamaulipas, México. Fue colectada en 1998, en una aislada localidad en el municipio de Hidalgo, cerca de los límites con el estado de Nuevo León. Se compara con E. bifida, E. walpoleana y E. strictiflora, a las que se asemeja en su morfología, pero las diferencias en el tamaño de la roseta, la forma y tamaño de las hojas, la presencia de mayor número de hojas de color vino cenizo, además de pedicelos largos de las flores individuales y el tamaño irregular de las brácteas y sépalos, ubican a $E$. rodolfi como una especie nueva perteneciente a la serie Angulatae.
\end{abstract}

\section{ABSTRACT}

Echeveria rodolfi is described as a new species from Tamaulipas, Mexico. It was collected in 1998 in an isolated locality of the municipality of Hidalgo near the boundary of the state of Nuevo León. It is compared with E. bifida, E. walpoleana and E. strictiflora, taxa similar in their morphology but, differences in the size of the rosette, in form and size of the leaves, presence of a large number of leaves of wine color, as well as of long pedicels of individual flowers, and the irregular size of bracts and sepals, place E. rodolfi as a new species belonging to the series Angulatae.

Durante las exploraciones botánicas efectuadas en los diferentes macizos montañosos de la Sierra Madre Oriental, encaminadas a inventariar la familia Cactaceae del estado de Tamaulipas y regiones adyacentes, se encontraron en los límites de los estados de Nuevo León y Tamaulipas algunos ejemplares pertenecientes al género Echeveria (Crassulaceae), los cuales no se pudieron identificar como alguna de las especies conocidas, por lo que la planta se propone como taxon nuevo para la ciencia.

Echeveria rodolfi Martínez-Ávalos J. G. et A. Mora-Olivo, sp. nov. Fig. 1.

Planta acaulis vel subacaulis, glabra. Foliorum rosula 16-23 (-30) cm diámetro, foliis crassissimis cymbiformibus, cinereo-vinaceis. Axis floralis $38 \mathrm{~cm}$ longus, bracteis alternis 

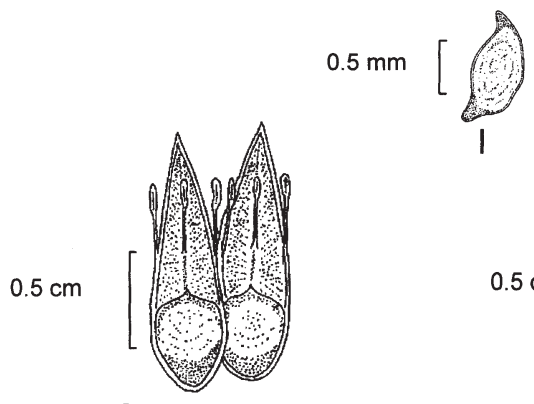

G
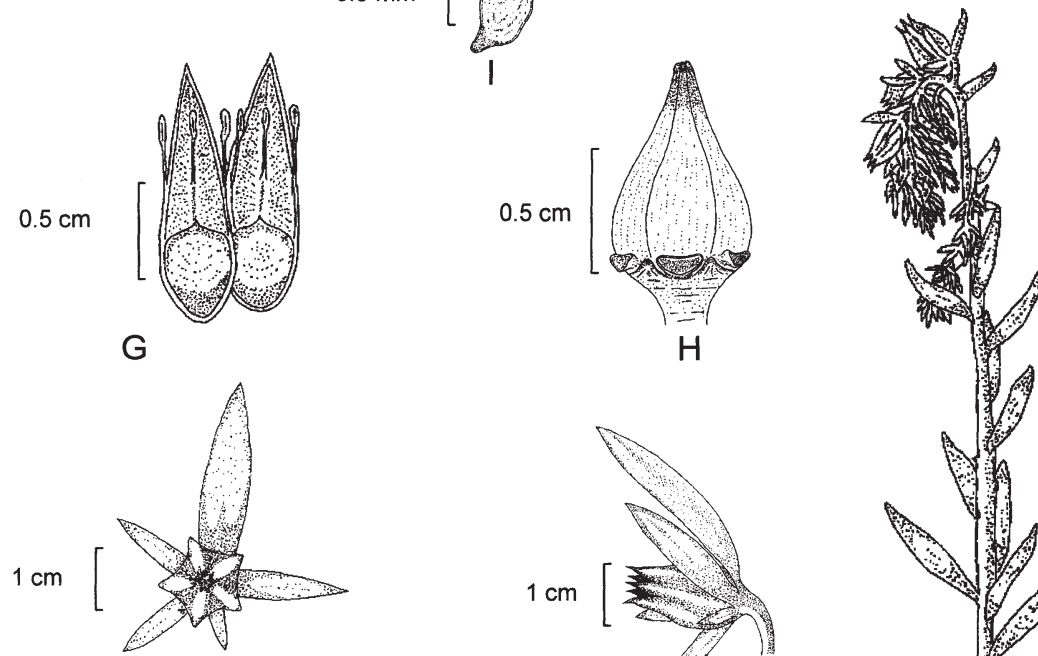

$\mathrm{H}$

$\mathrm{F}$
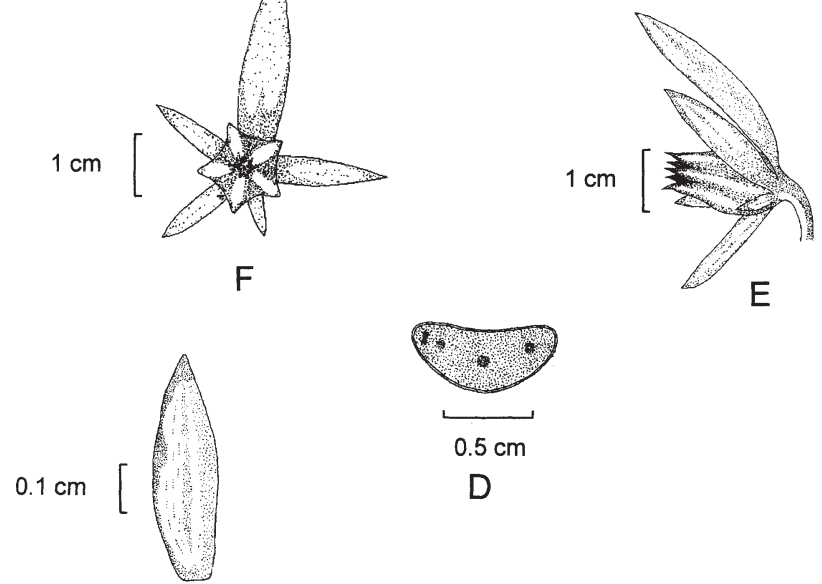

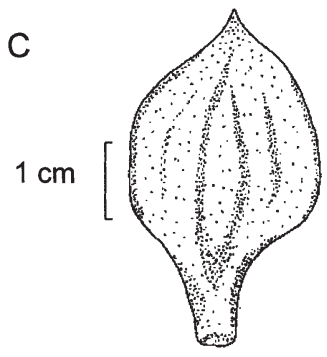

B
E

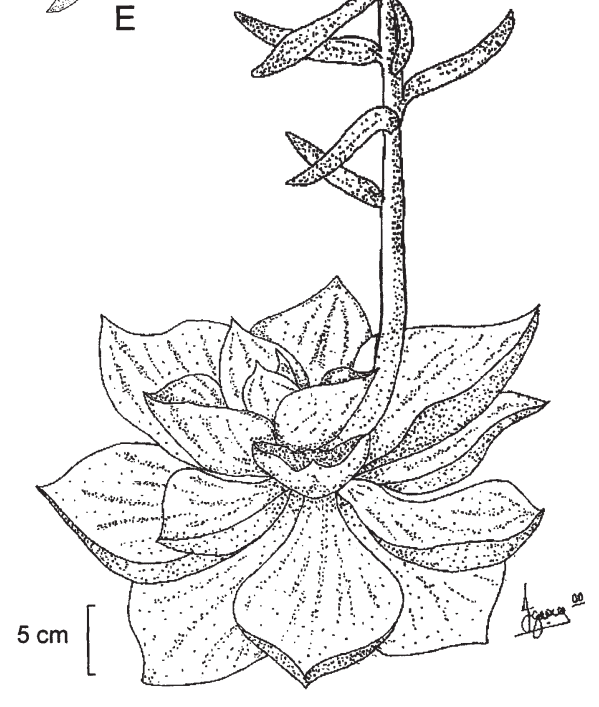

A

Fig. 1. Echeveria rodolfi A. Planta con inflorescencia; B. Hoja, vista adaxial; C. Bráctea, vista frontal; D. Corte transversal de una bráctea; E. Flor, vista lateral; F. Flor, vista frontal; G. Lóbulos; H. Carpelos y nectarios; I. Semilla. 
crassissimis, oblanceolatis, $6 \mathrm{~cm}$ longis, $0.9 \mathrm{~cm}$ latis. Inflorescentia 1-3 racemis nutantibus, circa 13 floribus in quoque racemo. Pedicelli $0.6 \mathrm{~cm}$ longi. Calyx 5 sepalis inaequalibus, longiore $1.6 \mathrm{~cm}$ longo, breviore $0.4 \mathrm{~cm}$ longo. Corolla tubularis, 5 lobis triangularibus, valde carinatis, roseo-aurantiacis. Semina falciformia vel cymbiformia, $0.8 \mathrm{~mm}$ longa, ferruginea.

Planta herbácea perenne, glabra, solitaria, acaule o con el tallo muy corto y cubierto por las hojas secas. Roseta de 16 a $23(-30) \mathrm{cm}$ de diámetro; hojas 24 o más, cimbiformes y extendidas, de 8.9 a $14.4 \mathrm{~cm}$ de largo y 6.1 a $8.5 \mathrm{~cm}$ de ancho en la parte media, de $0.5 \mathrm{~cm}$ de grosor, de color vino cenizo, las más jóvenes acanaladas con una costilla cerca de la base. Eje floral erecto, de $38(-45) \mathrm{cm}$ de largo, de $1.1 \mathrm{~cm}$ de grueso en la base, cilíndrico, de color verde blancuzco; brácteas 24 a 30, alternas, las inferiores de mayor tamaño que las superiores, dispuestas en un ángulo de $35^{\circ}$, de forma oblanceolada alargada, hasta de $6 \mathrm{~cm}$ de largo y $1.1 \mathrm{~cm}$ de diámetro, algo achatadas, de color verde a café oscuro; inflorescencia de 1 a 3 racimos unilaterales, de $7.1 \mathrm{~cm}$ de longitud, de color café oscuro cenizo; flores aproximadamente 13 por racimo, dispuestas en ambos lados del raquis; pedicelos de $0.6 \mathrm{~cm}$ de longitud; cáliz con 5 sépalos desiguales, libres, lineares, oblongos a oblanceolados, el mayor de $1.6 \mathrm{~cm}$ de largo por $0.4 \mathrm{~cm}$ de ancho, el menor de $0.4 \mathrm{~cm}$ de largo y $0.2 \mathrm{~cm}$ de ancho; corola tubular, ligeramente urceolada, con 5 lóbulos, de 1 a $1.2 \mathrm{~cm}$ de largo por $0.4 \mathrm{~cm}$ de ancho en la base, de forma triangular, aquillados, de color rosa naranja muy intenso en la parte ventral, formando un tubo; estambres 10, 5 epipétalos de $0.4 \mathrm{~cm}$ de largo, 5 episépalos de $0.06 \mathrm{~cm}$ de largo, de color crema, anteras de $0.2 \mathrm{~cm}$ de largo, de color amarillo oro; carpelos 5 , de $0.7 \mathrm{~cm}$ de largo por $0.3 \mathrm{~cm}$ de ancho; nectarios ampliamente deltoides de $1.8 \mathrm{~mm}$ de ancho, $0.6 \mathrm{~mm}$ de largo y $0.8 \mathrm{~mm}$ de grosor, blanquecinos. Semillas numerosas, falciformes a cimbiformes, de $0.8 \mathrm{~mm}$ de largo, de color café claro, testa con pequeñas papilas.

TIPO: México, Tamaulipas, municipio de Hidalgo, $42 \mathrm{~km}$ al oeste del poblado Santa Engracia, en los límites con el municipio de Zaragoza, Nuevo León; altitud 1900 m s.n.m.; bosque de pino y encino. 14 de octubre de 1998, J. G. Martínez-Ávalos 0819 (Holotipo: UAT; isotipos en proceso de reparto).

Distribución: Echeveria rodolfi sólo se ha encontrado en el municipio de Hidalgo en el filo de la Sierra Madre Oriental, en los límites de Tamaulipas y Nuevo León (Fig. 2). Su endemismo probablemente se restringe solamente a esta zona del noreste de México.

Habitat: De conformidad con las cartas topográficas del INEGI (Anónimo, 1998) la zona donde habita Echeveria rodolfi, se encuentra entre los 1800 a 2000 m s.n.m. En la región prevalece un clima templado con lluvias en verano $\left(C\left(\mathrm{w}_{2}\right)\right)$, la temperatura promedio anual oscila entre los 16 y $18^{\circ} \mathrm{C}$, de acuerdo con los datos de la estación climatológica del poblado Graciano Sánchez municipio de Hidalgo. El suelo es poco profundo, de color rojizo, del tipo de los litosoles, ricos en materia orgánica y con abundante fierro. La planta crece en pequeños manchones en el sotobosque de un pinar-encinar, en riscos de fuerte pendiente. Entre las especies acompañantes pueden destacarse: Pinus teocote, $P$. pseudostrobus, P. patula, Quercus rysophylla, Q. sartorii, Cornus sp., Carya ovata, Liquidambar styraciflua, Magnolia sp. Ferocactus hamatacanthus, Echinocereus pentalophus, Mammillaria glassii, Mammillaria rubrograndis. 
Acta Botanica Mexicana (2000), 52: 43-48
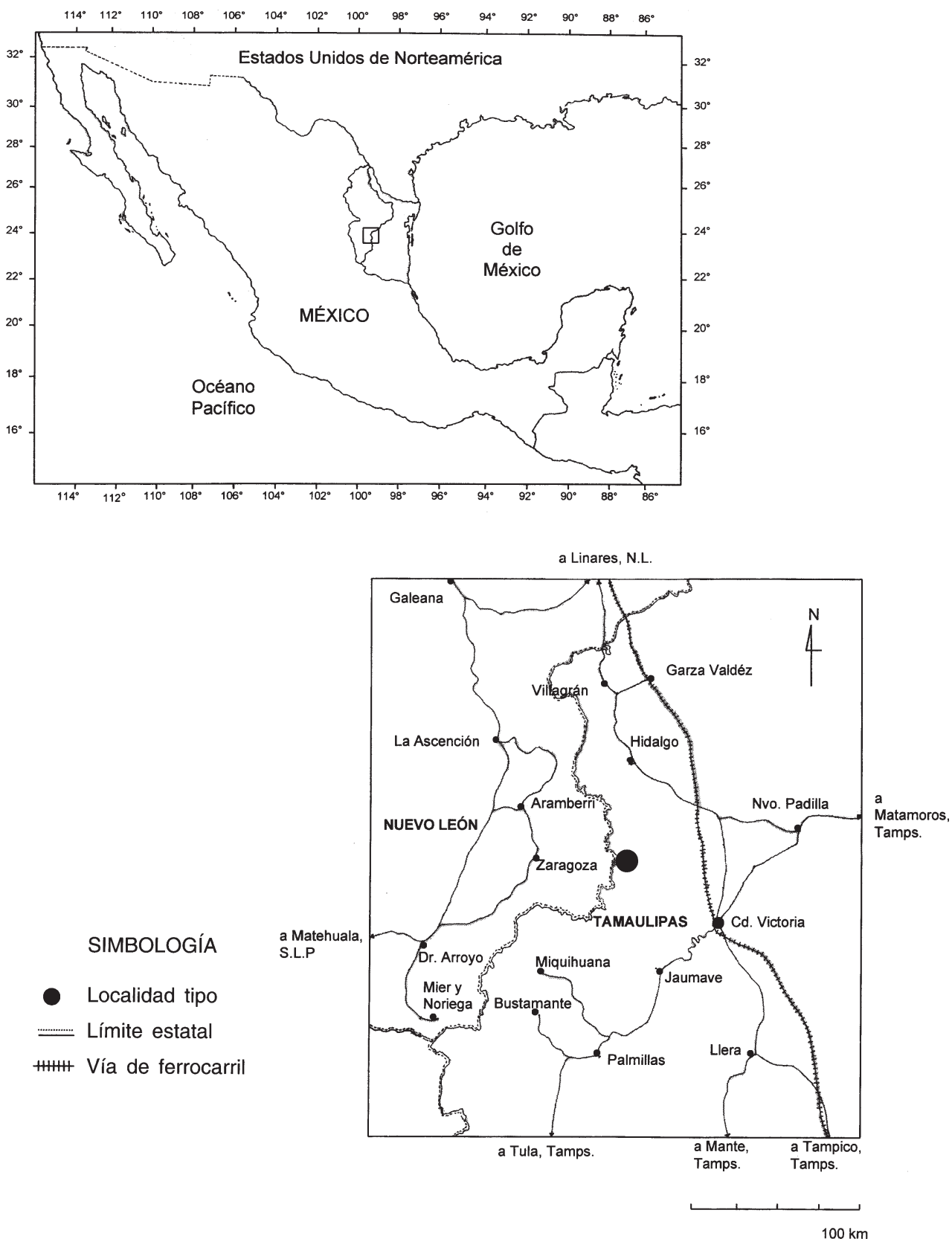

Fig. 2. Mapa de distribución de Echeveria rodolfi. 
Martínez-Avalos y Mora-Olivo: Una Nueva Especie de Echeveria del Estado de Tamaulipas

\begin{tabular}{|c|c|c|c|c|c|c|c|c|}
\hline 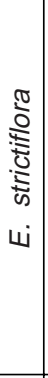 & 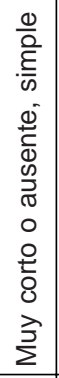 & 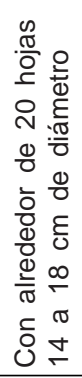 & 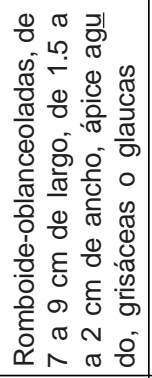 & 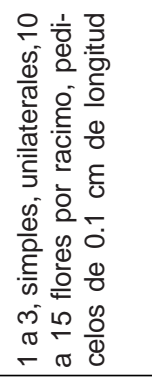 & 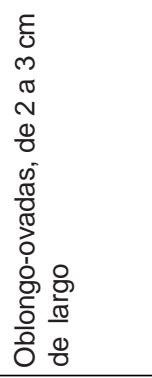 & 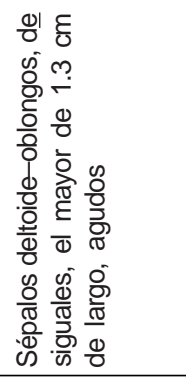 & 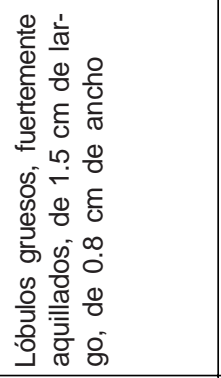 & 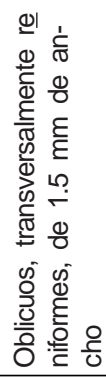 \\
\hline 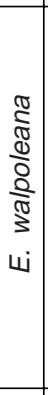 & 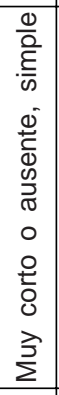 & 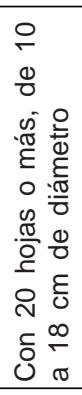 & 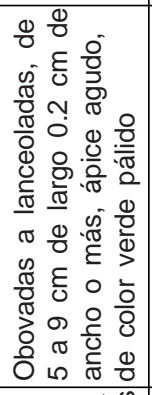 & 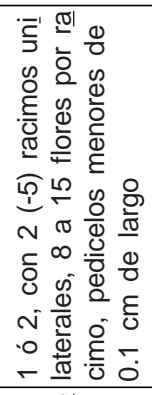 & 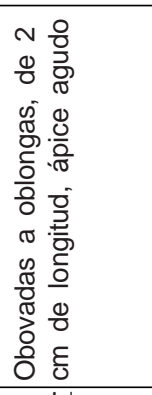 & 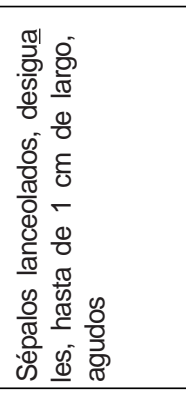 & 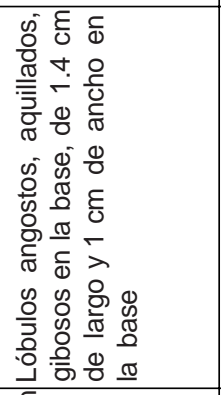 & 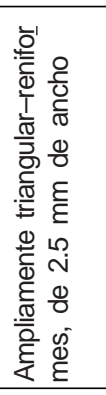 \\
\hline $\begin{array}{l}\frac{\pi}{2} \\
\stackrel{2}{0} \\
w\end{array}$ & $\begin{array}{l}\frac{0}{0} \\
\frac{\underline{E}}{\omega} \\
0 \\
\frac{0}{0} \\
0 \\
0 \\
\frac{0}{0} \\
\frac{E}{0} \\
\frac{0}{0} \\
0\end{array}$ & 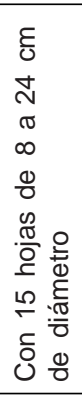 & 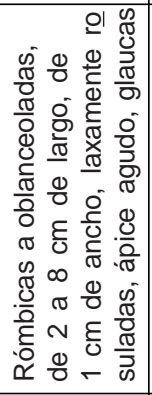 & 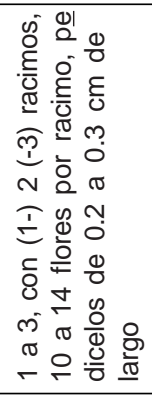 & 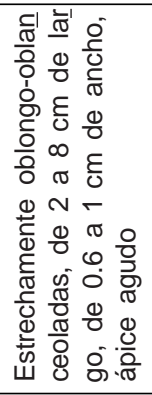 & 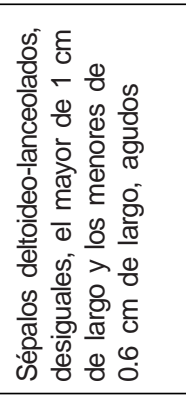 & 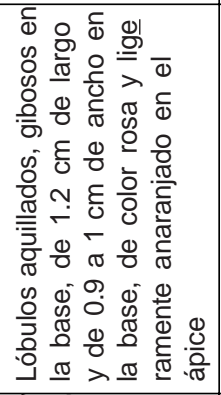 & 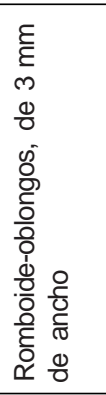 \\
\hline 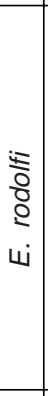 & 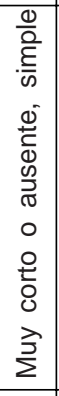 & 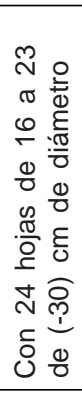 & 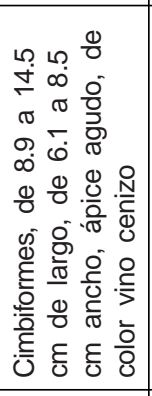 & 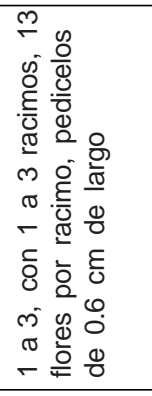 & 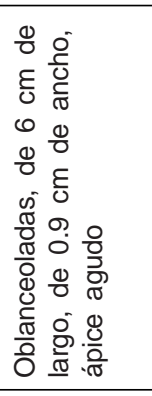 & 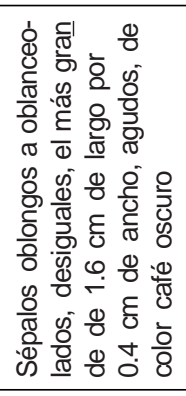 & 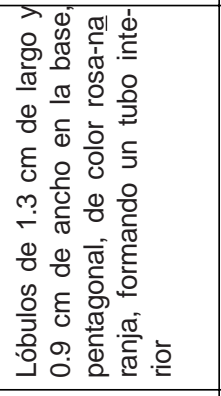 & 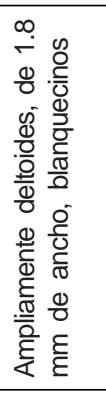 \\
\hline & $\frac{\stackrel{\circ}{\widetilde{\sigma}}}{\vdash}$ & $\begin{array}{l}\frac{\pi}{0} \\
\mathbb{0} \\
0 \\
\simeq\end{array}$ & $\frac{\frac{0}{0}}{\frac{\pi}{0}}$ & 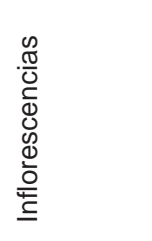 & 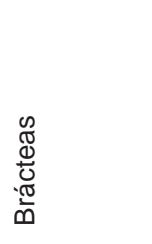 & $\frac{N}{\bar{N}}$ & $\begin{array}{l}\frac{\pi}{0} \\
\overline{0}\end{array}$ & 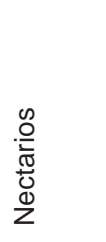 \\
\hline
\end{tabular}


Fenología: El período de floración ocurre durante los meses de abril y mayo. El tallo floral permanece por lo general hasta el siguiente año. Durante la época de sequías las rosetas disminuyen su tamaño, toman una coloración avinada muy ceniza, las hojas son más delgadas que durante la época de lluvias.

Etimología: El nombre de la especie se dedica a Rodolfo Martínez Gallegos, hijo del primer autor.

Siguiendo el criterio de Walter (1972), Echeveria rodolfi se ubica en la serie Angulatae E. Walter. Tiene semejanza con E. bifida Schlechtendal, E. walpoleana Rose y E. strictiflora A. Gray, aunque difiere de estas especies en el tamaño de la roseta, la forma y tamaño de las hojas y brácteas, la presencia de un pedicelo largo, además de un irregular tamaño de los sépalos. Asimismo, el color avinado cenizo de las hojas y el grosor de las mismas, permiten diferenciar de manera morfológica a esta especie con respecto a las demás (Cuadro 1). La localidad tipo de E. rodolfi, no corresponde con el área de distribución conocida de las especies anteriores.

\section{AGRADECIMIENTOS}

Se agradece a la Maestra Graciela Calderón de Rzedowski, al Dr. Jerzy Rzedowski y al Dr. Jesús Valdés Reyna sus interesantes comentarios y sugerencias sobre el escrito, asimismo al Dr. Fernando Chiang la traducción de la diagnosis al latín. Este trabajo se realizó gracias al apoyo económico de la Comisión Nacional para el Conocimiento y Uso de la Biodiversidad (CONABIO), a través de los proyectos P120 y H149.

\section{LITERATURA CITADA}

Anónimo. 1998. Cartas topográficas. Síntesis geográfica del estado de Tamaulipas. Instituto Nacional de Estadística, Geografía e Informática. Secretaría de Programación y Presupuesto. Aguascalientes.

Walter, E. 1972. Echeveria. California Academy of Sciences. San Francisco, California. 426 pp. 